\title{
Difficulty of Certain Sentence Constructions in Comprehension
}

\author{
Mineharu Nakayama, Shravan Vasishth, and Richard L. Lewis \\ The Ohio State University, University of Potsdam, and University of Michigan
}

Introduction

Native speakers of a language find some sentences more difficult to understand than others. The sources of difficulty vary: some are lexical (e.g., the use of unknown words), some are due to the lack of contextual information (e.g., no shared knowledge), and some are due to structural complexity. Here we will focus on the difficulties caused by one kind of structural complexity, center embeddings. The defining property of these structures is that a clause $\mathrm{X}$ appears within another clause $\mathrm{Y}$, with material from $\mathrm{Y}$ surrounding $\mathrm{X}$, i.e., the configuration $[\mathrm{Y} \ldots \mathrm{X} \ldots]$.

As discussed in the Introduction of this volume, the structure of Japanese, SOV word order, along with other morpho-syntactic characteristics such as no relative pronouns, agreement markers, and scrambling, inherently bring structural ambiguities while processing, in particular, when sentences are presented in writing (see Mazuka and Nagai 1995 and Nakayama 1999 for an overview of Japanese sentence processing). Examples of center embedded structures are relative clauses $(1 \mathrm{a}, \mathrm{b})$ and complement clauses $(1 \mathrm{c}, \mathrm{d})$.

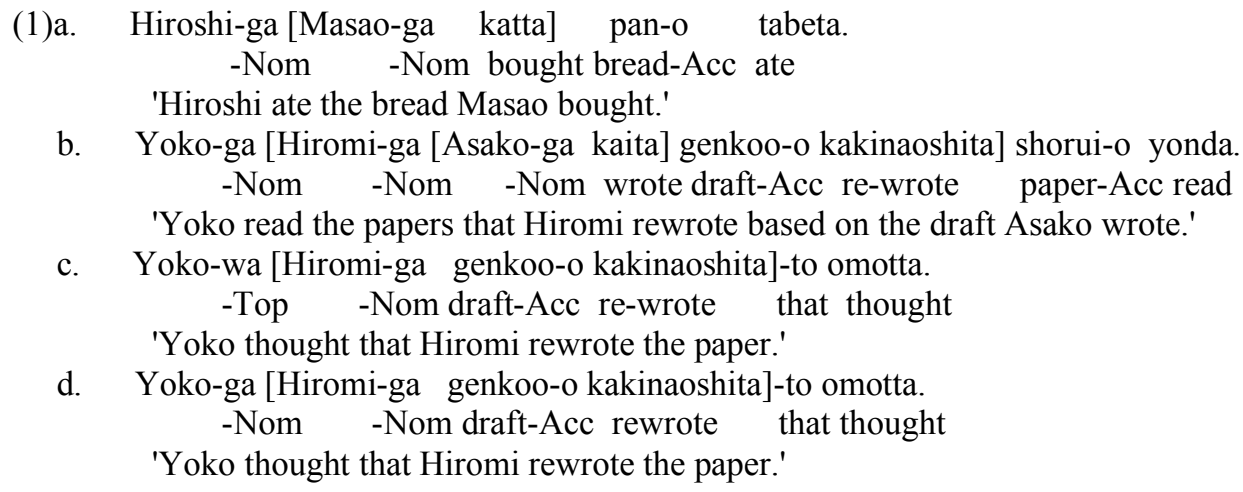

Sentence (1a) contains one relative clause modifying the matrix object while there are two recursively embedded relative clauses in (1b). Mazuka et al. (1989) find that the reading time per character for (1b) is longer than that for (1a), and (1b) is interpreted as more difficult. Since (1b) contains a relative clause within another relative clause, Mazuka and her colleagues' result suggests that the greater the embedding the harder it is to process the sentence (also see (Miller and Chomsky 1963)). Factors other than amount of embedding can also result in increased processing difficulty: sentences like $(1 \mathrm{c}, \mathrm{d})$ contain only one complement clause, yet (1d) is more difficult than those when the first NP is marked with -wa, as in (1c) (Lewis and Nakayama 2002). These center-embedded sentences are rather difficult to process, for instance, compared to simplex sentences and those with relative clauses in the beginning of the sentences.

This difficulty of comprehending center embeddings has been widely assumed to relate to working memory limitations (Babyonyshev \& Gibson 1995, 1999; Cowper, 1976; Gibson 1991, 1998, 2000; Gordon et. al. 2001, 2002; Lewis 1993, 1996, 1999; Lewis and Nakayama 2002; Miller, 1962; Miller \& Chomsky, 1963; Nakayama, Lee, \& Lewis, 2005; Uehara 1997, 2003; Uehara \& Bradley, 1996, 2002; Vasishth, 2003, among others; cf. Eady 1982). Though various accounts have been proposed, they can be largely grouped into two: a structural approach and a general psychological approach. The former tends to appeal to specific linguistic properties (e.g., counting heads, nodes, etc.) while the latter to more general properties (e.g., similarity-based interference). Given the space limitation, we will discuss one each of these two approaches below. It is important to remember that experimental results discussed here are from those of reading (i.e. visual presentation). This means that Japanese sentences were visually presented with appropriate kana and kanji in order to maintain a natural presentation for native speakers. 
As an example of the structural approach, we summarize Gibson's (2000) Dependency Locality Theory (DLT) here (see also Gibson 1998; Babyonyshev \& Gibson 1995, 1999; Grodner \& Gibson 2004; but see Uehara (1997) against Babyonyshev \& Gibson 1995). The DLT discusses two kinds of human computational resources in sentence parsing. They are in working memory needed for storage of the structure built up thus far and integration of the current word into the structure built up thus far. Storage costs are measured in Memory Units and Integration costs are measured in Energy Units.

Storage cost: 1 memory unit (MU) is associated with each syntactic head required to complete the current input as a grammatical sentence.

Structural integration cost: The structural integration cost associated with connecting the syntactic structure for a newly input head $h_{2}$ to a projection of a head $h_{1}$ that is part of the current structure for the input is dependent on the complexity of the computations that took place between $h_{l}$ and $h_{2}$. For simplicity, it is assumed that $1 \mathrm{EU}$ is consumed for each new discourse referent in the intervening region.

Discourse (simplified) processing cost (the cost associated with accessing or constructing the discourse structure for the maximal projection of the input word head $h_{2}$ ): 1 energy unit (EU) is consumed if $h_{2}$ is the head of a new discourse referent; 0 EUs otherwise.

These metrics can account for the difficulty differences between the subject and object relatives (e.g., Just and Carpenter, 1992, Grodner and Gibson 2004). Sentence (2a) contains a relative clause whose head (reporter) is extracted from the subject position (SR) and ( $2 \mathrm{~b}$ ) has a relative clause whose head is extracted out of the object position (OR). In (2a), who takes the highest storage cost while in (2b) the in the relative clause takes the highest storage cost and its cost is more than who in (2a).

(2)a. The reporter who attacked the senator disliked the editor.

$\begin{array}{llllllllll}2 & 1 & 3 & 2 & 2 & 1 & 1 & 1 & 0 & (\mathrm{MU})\end{array}$

b. The reporter who the senator attacked disliked the editor.
2
$\begin{array}{llll}1 & 3 & 4 & 3\end{array}$
$\begin{array}{lllll}1 & 1 & 1 & 0 & (\mathrm{MU})\end{array}$

Structural integration costs in these sentences are also different: the cost in (2a) is less than that in (2b).

(2)a'. The reporter who attacked the senator disliked the editor.

\section{$1 \mathrm{EU}$}

b'. The reporter who the senator attacked disliked the editor.

$$
3 \text { EUs }
$$

When the relative clause verb attacked is read, its integration cost is $1 \mathrm{EU}$ in (2a) whereas it is 3EUs in (2b). Structural integration complexity depends on the distance/locality between the two elements being integrated. When an OR is embedded in another OR as in (3), the integration cost of attacked becomes much higher, i.e., 7EUs.

(3) The reporter who the senator who John met attacked disliked the editor.

$$
7 \text { EUs }
$$

Since OR sentences take more storage and structural integration costs than SR sentences, they are more difficult to comprehend. Grodner and Gibson (2004) provide further evidence from English in support of DLT.

Although structural accounts like Gibson's can correctly account for the difficulty of OR sentences in English, Hirose and Inoue (1998) argue that OR is not an inherently difficult construction to 
process. They tested the following three types of sentences and found different reading times at the head noun position depending on the thematic roles of the head noun.

(4)a. Yamaoka-ga kakushiisan-o anote konote-de sagashidashita moguri-no bengoshi-ni -Nom hidden fortune-Acc after great effort discovered back-street lawyer-Dat yamunaku azuketa. (OR, Theme, but ambiguous between Agent or Theme) unwillingly entrusted 'Yamaoka unwillingly entrusted his hidden fortune to the unlicensed lawyer who he discovered after great effort.'

b. Yamaoka-ga kakushiisan-o anote konote-de sagashidashita moguri-no kashikinko-ni -Nom hidden fortune-Acc after great effort discovered back-street safe-Dat yamunaku azuketa. (OR, Theme) unwillingly entrusted 'Yamaoka unwillingly entrusted his hidden fortune to the unlicensed safe that he discovered after great effort.'

c. Yamaoka-ga kakushiisan-o anote konote-de sagashidashita moguri-no bengoshi-ni -Nom hidden fortune-Acc after great effort discovered back-street lawyer-Dat yamunaku ayamatta. (SR, Agent) unwillingly apologized 'Yamaoka unwillingly apologized to the unlicensed lawyer who discovered his hidden fortune after great effort.'

The sentences in (4) are different in underlined parts. (4a) and (4b) are OR constructions while (4c) is a SR construction. (4a) and (4c) are different only at the final verb position. The head noun bengoshi 'lawyer' is ambiguous between Agent and Theme of the relative verb sagashidashita 'discovered', but the final verbs azuketa 'entrusted' and ayamatta 'apologized' make it Theme and Agent in (4a) and (4c), respectively. On the other hand, (4b) is the same OR as (4a), but different only in the head noun: it contains kashikinko 'safe' instead of 'lawyer'. However, this inanimate noun allows itself to be read as Theme of 'discovered'. There is no thematic ambiguity as in (4a) while the head noun is being read. They found the head noun in (4b) was read significantly faster than that in (4a) and there were no significant differences between (4a) and (4c) at the head noun position. This suggests that the difficulty of OR changes depending on the detectability of the thematic role of the head noun. A structural account like Gibson's may need to be adjusted considering thematic differences.

General Psychological Approach

Another approach to the difficulty of particular type of sentences appeal to more general psychological factors, e.g., similarity-based interference. For instance, consider the following center-embedded sentences.

(5)a. That the food that John ordered tasted good pleased him. (Cowper, 1976; Gibson, 1991)

b. The salmon that the man that the dog chased smoked fell off the grill.

Although (5a) involves two levels of center embedding of sentential structures, it does not cause the comprehension difficulty that $(5 \mathrm{~b})$ does. Increasing center embedding certainly increases difficulty, but a metric based solely on the amount of center embedding does not account for many difficulty contrasts in English and other languages. Another claim is that self-embedding (i.e., increasing the similarity of the embedded constituents) increases difficulty, and making constituents more distinct or dissimilar in some way helps processing (e.g., Bever, 1970; Miller and Chomsky, 1963; Kuno, 1974).

Similarity-based interference is a principle that applies to working memory independently of language processing. Lewis (1996) reviews evidence for a range of working memory types subject to selective, type-specific interference, including verbal, spatial, odor, kinesthetic, and sign language. The robust result across domains is that when to-be-remembered items are followed by stimuli that are similar along some dimensions, the original items are more quickly forgotten. Lewis $(1993,1996)$ hypothesize that similarity-based interference is a general principle that applies to syntactic working memory as well. Lewis described a computational model that embodies retroactive, type-specific syntactic 
interference, and accounts for a range of cross-linguistic data on difficult center-embeddings. The model posited a simple buffer that could maintain no more than two constituents of a particular syntactic type. Consider the comprehensible Japanese construction in (6) below (Lewis, 1993):

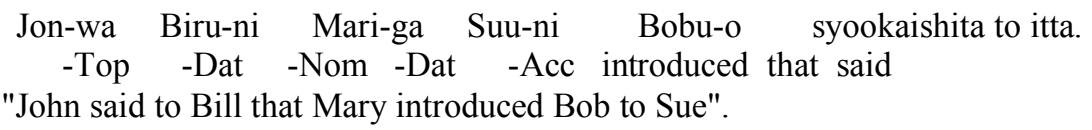

Sentences like (6) do not cause the difficulty associated with (5b), despite stacking up five NPs. A crucial difference is that (6) requires buffering no more than two NPs of any particular syntactic function: at most two subjects, two indirect objects, and a direct object. What this theory amounts to is adding "syntactic" to the list of immediate memory types that exhibit type-specific interference and decreased performance with increased similarity. Just as there is the well-known phonological similarity effect (e.g., Baddley, 1966), there is also a "syntactic similarity effect", and one way this effect manifests itself is difficulty with centerembedding. For instance, as mentioned above, Lewis and Nakayama (2002) found that a sentence like (1d) is more difficult than (1c) (see also Nakayama, Lee, and Lewis, 2005). Since (1d) contains two - ga marked NPs, while (1c) has only one, the former is more difficult than the latter. Furthermore, it was found that a sentence like (7b) is more difficult than a sentence like (7a). This is because two - $g a$ marked NPs are adjacent in (7b), while they are not in (7a). Two items with the same grammatical functions must be distinguished based on serial position, and are therefore subject to positional confusions which is maximized when they are adjacent.

(1)c. Yoko-wa [Hiromi-ga genkoo-o kakinaoshita] to omotta.

-Top -Nom draft-Acc re-wrote that paper-Acc read

'Yoko thought that Hiromi rewrote the paper.'

d. Yoko-ga [Hiromi-ga genkoo-o kakinaoshita] to omotta.

-Nom -Nom draft-Acc re-wrote that paper-Acc read

'Yoko thought that Hiromi rewrote the paper.'

(7)a. Yoko-ga Kaoru-ni [Hiromi-ga kookoosei-o shinsasuru] to yakusokushita.

-Nom -Dat -Nom HS student-Acc examine that promised

'Yoko promised Kaoru that Hiromi would examine the HS student.'

b. Yoko-ga [Hiromi-ga Kaoru-ni sakka-o shokaishita] to kizuita.

-Nom -Nom -Dat writer-Acc introduced that noticed

'Yoko noticed that Hiromi introduced the writer to Kaoru.'

Based upon Uehara and Bradley's (1996) finding on Korean, Lewis and Nakayama (2002) conclude that the processing difficulty of $g a-g a$ sentences compared to wa-ga sentences is due to the effects of syntactic similarity. However, Vasishth (2003) presents evidence for the effects of morphophonemic similarity in Hindi. Thus, it is not clear whether similarity at the morphophonemic level is a source of difficulty or that at the syntactic level. Nakayama, Lee, and Lewis (2005) investigate this point. Since Japanese has only one nominative case marker, it cannot be tested in Japanese. So Korean was employed, a language which is structurally similar to Japanese, but different in that it has two morphophonemic distinct nominative case markers, $-k a$ and $-i$. Vocalic ending nouns take $k a$ as a nominative case marker while consonantal ending nouns take $i$. The processing difficulty of the six sentence types in (9) were tested by using a magnitude estimation moving window task (see Bard, Robertson, \& Sorace (1996) for the magnitude estimation task, and Cohen, MacWhinney, \& Provost (1993) on a moving window program called Psyscope). Each test sentence contained three two-syllable-nouns and two verbs. NP1 and NP2 were proper nouns while NP3 was a common noun. All nouns and verbs were controlled in terms of their familiarity ratings. NP1 had three types of markers, topic -num, nominative $-k a$, and nominative $-i$, whereas NP2 had two variations of nominative case markers $-k a$ and $-i$.

(9) $[\mathrm{NP} 1-\mathrm{nun} / \mathrm{ka} / \mathrm{i}[\mathrm{NP2}-\mathrm{ka} / \mathrm{i} \mathrm{NP3}-\mathrm{lul} \mathrm{V}] \mathrm{V}]$

e.g., Euncwu-nun/-ka/Huisen-i Youngay-ka/Misun-i kyoswu-lul chacawass-tako kiekhayssta.

'Encwu/Huisen remembered that Youngay/Misun had visited the professor.' 
It was found that topic-nominative sentences were significantly easier from nominative-nominative sentences, and the same nominative sequences $(k a-k a$ and $i-i)$ were significantly more difficult than dissimilar sequences. The former finding was the same as in Japanese and the latter was consistent with the findings in Vasishth (2003). This seems to suggest that the difficulty of two nominative NPs in Japanese, e.g., (1d), could be due to the effects of both morphophonemic and syntactic similarity interference. Although much works remains to be done, this approach provides the opportunity to establish close ties between sentence processing theory and accounts of serial order in other more general theories of memory. See also Gordon, et al. (2001), Gordon, et al. (2002), Uehara (2003), and Uehara \& Bradley (2002).

\section{Concluding Remarks}

The difficulty of comprehending various constructions, especially, center-embedding constructions, seems related to working memory limitations. Among those accounts that appeal to working memory limitations, we have looked at two approaches, structural and general psychological approaches. The former theory is defined in terms of linguistic properties (the notion of discourse referents, and argument-head relations) while the latter is defined in within the framework of cognitive psychology research. Although the second approach is arguably preferable to the first due to its greater generality, it is still an open question whether the more general approach can explain a wider range of phenomena and better cross-linguistic coverage. Recently Vasishth and Uszkoreit (2004) have found that the RC difficulty in German coincides with the frequency of self-center embedding clause type. Since this frequency account is not attested in Japanese, it needs to be examined. More research is ongoing.

\section{References}

Baddley, A. D. (1966) Short-term memory for word sequences as a function of acoustic, semantic, and formal similarity. Quarterly Journal of Experimental Psychology 18, 362-365.

Bard, E. G., D. Robertson, \& A. Sorace (1996) Magnitude estimation of linguistic acceptability. Language 72.1.32-68.

Bever, Thomas G. (1970). The cognitive basis for linguistic structures. In J. R. Hayes (ed), Cognition and the Development of Language . 279-362. New York: Wiley.

Babyonyshev, M. \& Gibson, E. (1995). Processing overload in Japanese. In C. T. Schutze, J. B. Ganger, and K. Broihier (eds), Papers on Language Processing and Acquisition: MIT Working Papers in Linguistics 26. 1-36. Cambridge, MA: Department of Linguistics, Massachusetts Institute of Technology.

Babyonyshev, M. \& E. Gibson (1999) The complexity of nested structures in Japanese. Language 75.3, 423-450.

Chomsky, Noam. (1959). On certain formal properties of grammars. Information and Control. 2:137-167.

Cohen, J. D., B. MacWhinney, M. Flatt, \& J. Provost (1993) PsyScope: A New Graphic Interactive Environment for Designing Psychology Experiments. Behavioral Research Methods, Instruments \& Computers 25.2, 257-271.

Cowper, Elizabeth A. (1976). Constraints on Sentence Complexity: A Model for Syntactic Processing. Doctoral dissertation, Brown University.

Eady, Stephen. 1982. Is center-embedding a source of processing difficulty? ms. University of Connecticut.

Gibson, Edward A. (1991). A Computational Theory of Human Linguistic Processing: Memory Limitations and Processing Breakdown. Doctoral dissertation, Carnegie Mellon University.

Gibson, Edward A. (1998). Linguistic complexity: locality of syntactic dependencies. Cognition 68: 1-76.

Gibson, E. (2000) Dependency locality theory: A distance-based theory of linguistic complexity. In A. Marantz, Y. Miyashita, and W. O'Neil (eds.), Image, Language, Brain: Papers from the first mind articulation project symposium. Cambridge: MIT Press.

Gordon, P., C. Hendrick, \& M. Johnson (2001) Memory interference during language processing. Journal of Experimental Psychology: Learning, Memory \& Cognition 27.6, 1411-1423.

Gordon, P., C. Hendrick, \& W. H. Levine (2002) Memory-load interference in syntactic processing. Psychological Science 13.5, 425-430.

Grodner, Daniel J., \& Edward. A. F. Gibson (2004) Consequences of the serial nature of linguistic input for sentential complexity. Cognitive Science (in press). 
Hirose, Yuki \& Atsu Inoue (1998) Ambiguity of reanalysis in parsing complex sentences in Japanese. In D. Hillert (ed.), Syntax and Semantics 31: Sentence Processing: A Crosslinguistic Perspective. 71-93. New York: Academic Press.

Just, Marcel A. \& Patricia A. Carpenter (1992) A capacity theory of comprehension: Individual differences in working memory. Psychological Review 99, 122-149.

Kuno, Susumu. 1974. The position of relative clauses and conjunctions. Linguistic Inquiry 5, 117-136.

Lewis, Richard L. (1993). An Architecturally-based Theory of Human Sentence Comprehension. Doctoral dissertation, Carnegie Mellon University.

Lewis, R. L. (1996) Interference in short-term memory: The magical number two (or three) in sentence processing. Journal of Psycholinguistic Research 25.1, 93-115.

Lewis, Richard L. (1998). Interference in working memory: Retroactive and proactive interference in parsing. ms. The Ohio State University.

Lewis, R. L. \& M. Nakayama (2002) Syntactic similarity effects of embeddings in Japanese. In M. Nakayama (ed.), Sentence Processing in East Asian Languages. 85-110. Stanford: CSLI.

Mazuka, R., Itoh, K., Kiritani, S., Niwa, S., Ikejiri, K. \& Naitoh, K. (1989). Processing of Japanese garden path, center-embedded, and multiply left-embedded sentences. Annual Bulletin of the Research Institute of Logopedics and Phoniatrics, 23, 187--212. University of Tokyo.

Mazuka, R. \& N. Nagai eds. (1995), Japanese Syntactic Processing . Hillsdale, NJ: Lawrence Erlbaum Associate.

Miller, George A. (1962). Some Psychological Studies of Grammar. American Psychologist 17.748-762.

Miller, George A. and Noam Chomsky. (1963). Finitary Models of Language Users, in D. R. Luce, R. R. Bush and E. Galanter, eds., Handbook of Mathematical Psychology Vol.II. New York: John Wiley.

Nakayama, Mineharu. (1999). Sentence Processing, in N. Tsujimura, ed., The Handbook of Japanese Linguistics. 398-424. Boston: Blackwell.

Uehara, Keiko. (1997). Judgments of processing load in Japanese: The effect of NP-ga sequences. Journal of Psycholinguistic Research, 26.2, 255-63.

Uehara, K. and D. Bradley (1996) The effect of - $g a$ sequences on processing Japanese multiply centerembedded sentences. In B. Park and J. Kim (eds.), Language, Information and Computation. Seoul: Kyung Hee University.

Uehara K. and D. Bradley (2002) Center-embeddeing problem and the contribution of nominative case repetition. In M. Nakayama (ed.), Sentence Processing in East Asian Languages. 257287. Stanford: CSLI.

Uehara, Keiko (2003) Center-embedding and nominative repetition in Japanese sentence processing. Doctoral dissertation. The City University of New York.

Vasishth, S. (2003) Working Memory in Sentence Comprehension: Processing Hindi center-embeddings. Garland Press (Routledge): New York.

Vasishth, S. \& Hans Uszkoreit (2004) Self-center embeddings revisited. Poster presented at AMLaP Conference. 